\begin{tabular}{|c|c|}
\hline \multirow{3}{*}{ 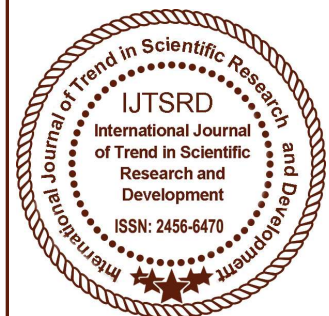 } & $\begin{array}{l}\text { International Journal of Trend in Scientific } \\
\text { Research and Development (IJTSRD) }\end{array}$ \\
\hline & International Open Access Journal \\
\hline & ISSN No: 2456 - 6470 | www.ijtsrd.com | Volume - 2 | Issue -4 \\
\hline
\end{tabular}

\title{
Case Study of Augmented Reality Applications in Medical Field
}

\author{
Vaishnavi D. Deolekar, Pratibha M. Deshmukh ${ }^{2}$ \\ ${ }^{1}$ Student, ${ }^{2}$ Professor \\ Bharati Vidyapeeths, Institute of Management \& Information Technology, \\ Navi Mumbai, Maharashtra, India
}

\begin{abstract}
Computerized applications are used at greater extent that helps the training in medical field. Augmented reality applications possess an interactive virtual layer on top of reality. The use of augmented reality applications acts as a boon to medical education because they combine digital parts with the practical learning environment. The aim of this research is to investigate the scope of augmented reality applications in medical professionals training [1]
\end{abstract}

This technology is different from virtual reality, in which the user is immersed in a virtual world generated by the computer. The AR system brings the computer into the user world by augmenting the real environment with virtual objects. [2]

In Augmented Reality, physical and artificial objects are mixed together in a hybrid space where the user can move without constraints. This paper aims to provide information of current technologies and benefits of augmented reality and to describe the benefits and open issues. [3]

Keywords: Computer augmented environment; User interfaces; Head-mounted display; augmented reality; surgery

\section{INTRODUCTION}

The medical zone is a zone in which complicated learning occurs. Complicated learning involves understanding complicated physiological systems, developing robust proficiency and acquiring the collective art required in comprehensive medical practice. It involves mastery of knowledge that enables the individual to perform vocational activities to the standards expected in the professional environment. This requires sufficient opportunity to practice and the potentiality to experience all possible contrasts in contexts and conditions in order to reach the expert level.

Educational technology about $\mathrm{AR}$, is favourable for facilitating relevant learning ; furthermore it may offer managerial benefits because:

the manual training environment may be very alike to, if not the consistent, as the expert work environment

the augmented (virtual) part may imagine the indistinguishable and replicate relevant 3D [7], and other aspects of the real world task

the AR learning environment may provide the necessary alterations in the training task including association which supports valid learning

AR systems are

1. Combine real and virtual

2. Are interactive in real time

3. Register in three dimensions.

Which produces Mixed Reality includes augmented reality (AR) and augmented virtuality (AV).

\section{TECHNOLOGIES AND ARCHITECTURES OF AR SYSTEMS}

AR technologies were immediately identified as a valuable tool to bring patients and their medical data into the same space. The potential of AR in medical applications was foreseen by Steinhaus (an Austrian mathematician) in 1938. 
The use of AR in surgery is strictly related to the display technology the surgeon opts for: computer generated assets can be directly overlapped onto the operating microscope or can be displayed over a monitor (augmented endoscopes can be considered as a particular case of monitor-based AR).

Fig. 2 shows an example of medical application: the patient's medical record is shown and the radiography image is overlapped to the face with precise positioning.

The projection of assets directly on the patient is apart from a particular visualization technology, but it involves very complex setups, which need accurate calibrations. Much more than other application fields, $\mathrm{AR}$ in medicine has to overcome three main issues: tracking precision, misperception and interaction with synthetic data.

On the other hand, medical AR generally involves very limited and controlled working indoor volumes and current tracking systems are able to provide the required precision under these conditions. The interaction issue is more generally related to user interface design problems; for instance, a / surgeon cannot interact with assets by using touch, therefore natural and multimodal user interfaces have to be implemented. Unfortunately, these alternative input modes can introduce robustness issues, which have to be taken into account when safety-critical systems such as medical ones are designed.

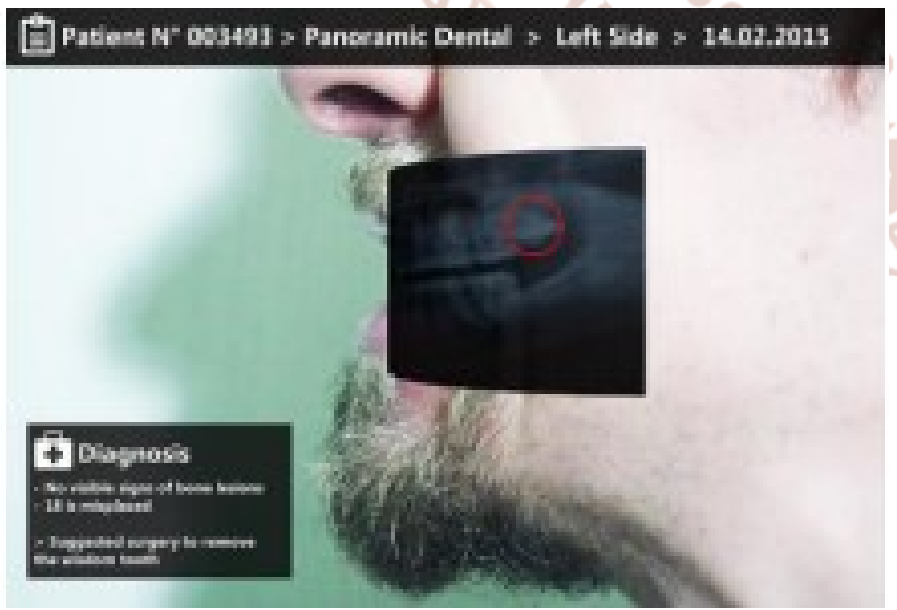

Fig. 2 Medical application for dental surgery.

The application shows the medical record for a patient, overlapping the radiography image to the face; points of interest are highlighted as well as doctor's annotations.

\section{APPLICATIONS}

\section{Anatomy 4D}

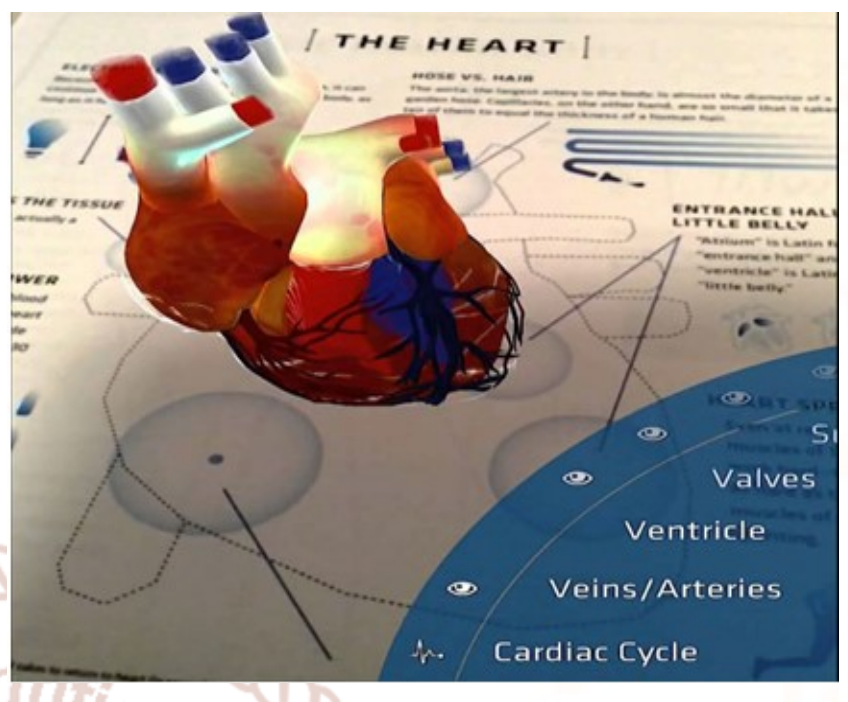

This is again an app developed for the educational purposes for the medical colleges. This app gives an interactive 4D experience of human anatomy to teachers, medical professionals, and students of all levels. [6]

\section{Diagnostic and Treatment Applications of AR}

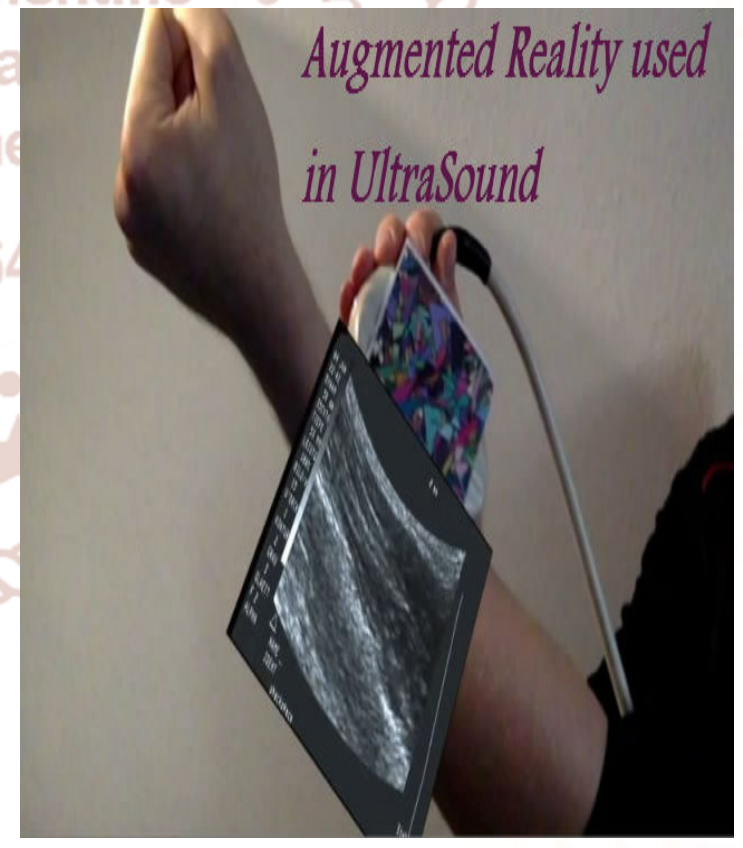

It has given surgeons an ability to retrieve information about the patient obtaining the treatment much rapidly. This facility has actually helped the surgery procedures to the maximum as the important details can be made visible all the time without diverting their attention from patient to screens. [6] 
3. Eye Decide Application

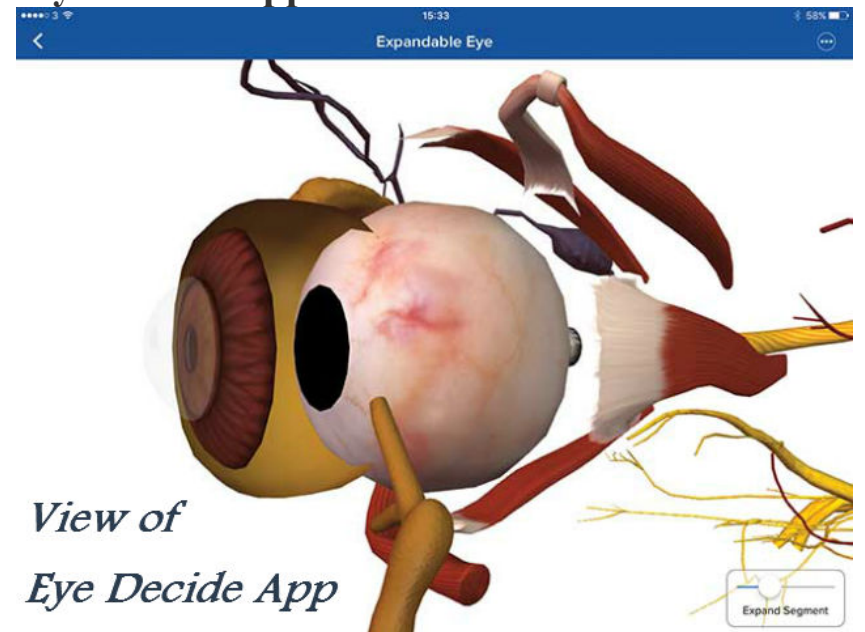

The application can demonstrate the impact of Cataract or AMD and thus help patients understand their symptoms and medical state in a better way. It can also help them understand the long-term effects of their lifestyle on their health. [6]

\section{VIPAAR}

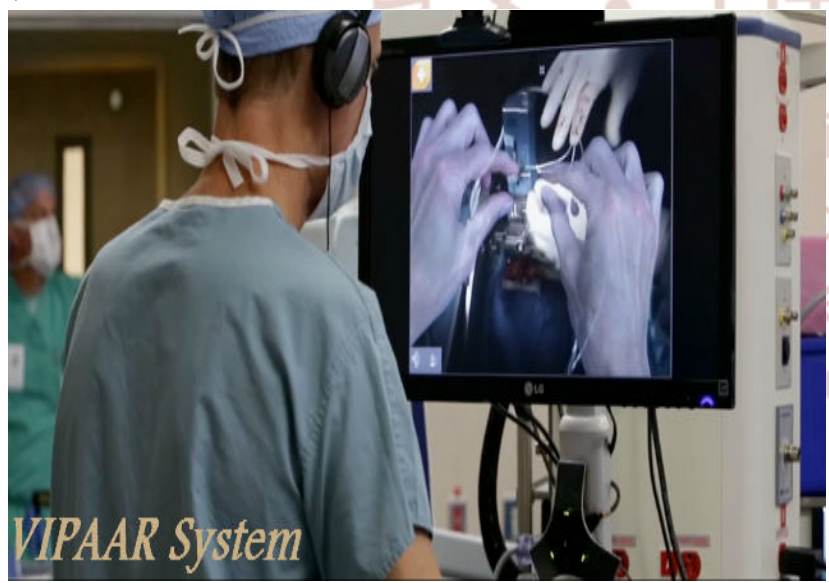

VIPAAR is short for Virtual Interactive Presence and Augmented Reality for remote surgical assistance. It is a video support solution, which helps a remote surgeon to operate a patient via the surgeon on the site wearing Glass and point and guide. [6]

\section{AccuVein}

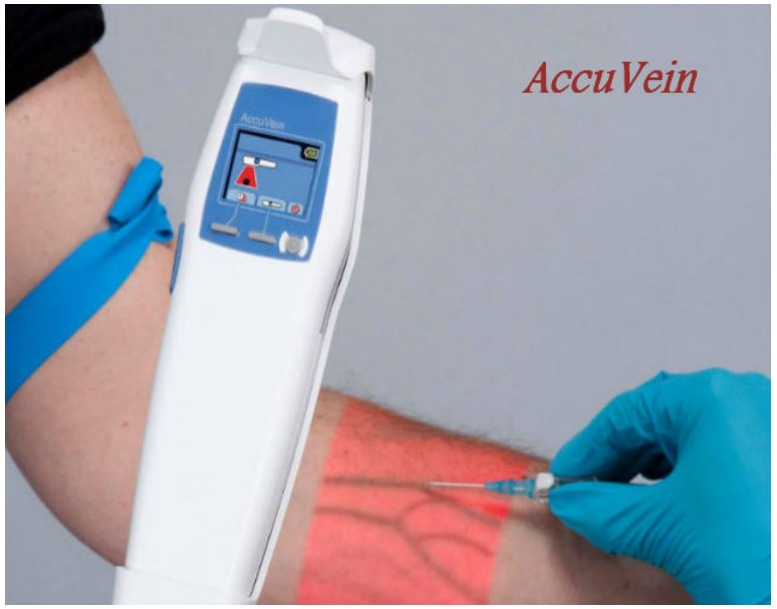

AccuVein, a company that makes the work of nurses and patients easy by using the AR Technology. AccuVein is a handheld scanner that scans over skin and guides nurses and doctors where veins and their valves and bifurcations are situated in patients bodies. [6]

\section{RESEARCH METHODOLOGY}

The study is based on exploratory research. Secondary data is collected from various sources such as books, web, broadcasting and articles.

\section{STRENGTH OF AUGMENTED REALITY [7]}

\section{Critical Data in plain view and hands -free gesture technology}

Nowadays, computer devices helps the doctors to easily record the information of patient. The process of maintaining the patient information has been changing with technologies like gesture technology paired with AR glasses that allows medical professionals to see patient information projected in front of them and then record information with hand and motions.

\section{Taking or transfusing blood}

Elderly patients and infants have very small veins due to which blood collection from their bodies is not so easy. Companies like Evena developed an AR solution that makes doctors and nurses visible the veins of their patients see easily on their arm, which makes it easy for blood tests. The smart glasses map out the veins on the outside of the patients arm so that the process of blood collection is easier for the patient.

\section{Ultrasounds}

The AR software companies have developed transportable ultra sound technology. This technology helps the technicians and patients to view the ultra sound image through smart glasses. A transportable ultra sound can be very useful while travelling where portability is important.

\section{Spinal Surgery}

The spinal surgery sometimes is a long process. The AR technology is trying to reduce the time for surgery and the result of it. Israeli-based company Augmedics created a technology that displays spinal information on the spine of the patient so that doctors can monitor the patient throughout and have the spinal information as needed. Surgeons wear 
a head-mounted display that allows the surgeons to see through the patients' skin as if they have X-ray vision.

\section{WEAKNESSES OF AUGMENTED REALITY} Social Rejection

Though AR is modern but it may become difficult for the people to adapt it. It may not. They may not trust the results of the applications.

\section{Poor Experience}

The concept is useful but when it is used it might seem to be lacking somewhere. Its poor resolution, User interface, \& the actual experience might not reach upto the user's expectations.

\section{Digital Fatigue}

The combination of digital and physical worlds is just too much beyond the expectation. The people have been surrounded by their mobile phones, television but sometimes its better to visible the virtual information directly being existed.

\section{Legal}

AR companies are unable to track the legal issues while using it at scale. The issue of privacy and safety lead to huge regulation, making it difficult for AR development.

\section{$>$ Security}

AR hackers directly implant harmful content into applications via advertising. Many attackers control the AR channels directly. The users may click on such ads that leads to the website server and may result into malicious attack. There are also network attackers who can monitor interactions between AR browsers and users. [10]

\section{THREATS}

\section{Invades Privacy}

AR can be prone to many people one to data hacks which is dangerous as we are unaware of of how much data is presented in virtual environment. Many people might fear that the way in which the information is accessed by the authorities which may result in the attack..[9]

\section{$>$ Hampers Interaction with Real-World}

AR may provide new innovative ways for people to communicate, but there could also be possibility that people might lose their social life through reduced human interaction. [9]

\section{CONCLUSION}

Augmented reality applications that have supported combination learning has resulted in public interest. The use of AR technology can be proved to be great help for medical professionals which result in less risk of the patients treatment. People might find of great help which would make their life easy. It can help a lot in development of innovative ideas.

\section{RECOMMENDATION}

Till now the extent of help the applicator provides is remarkable. We believe that it can be more useful if capable of transferring to the user. The application can suggest the solutions for the problems faced. If the user has can get the direct access to the information provided by application it would prove to be of great help.

\section{REFERENCES}

1. https://www.ncbi.nlm.nih.gov/pmc/articles/PMC5 009168/

2. https://ieeexplore.ieee.org/document/677169/

3. https://pdfs.semanticscholar.org/7b78/7a261a82e5 488fc972d2b6541f778c81ed78.pdf

4. https://www.ncbi.nlm.nih.gov/pmc/articles/PMC4 $152469 /$

5. https://blogs.systweak.com/2017/05/applicationsof-augmented-reality-in-the-field-of-medicalsciences/

6. "https://hitconsultant.net/2017/08/04/augmentedreality-medical-world/

7. http://www.behindthespin.com/features/advantage s-disadvantages-of-augmented-reality

8. http://www.humavox.com/blog/pros-consaugmented-virtual-reality/

9. https://haptic.al/augmented-realitys-biggestthreats-3f4726a3608

10. https://yourstory.com/mystory/21b7cafae1-thesecurity-risks-with-augmented-reality-and-waysto-combat 